\title{
BEOBACHTUNGEN UND VERSUCHE UBER DAS SINNESLEBEN UND DIE INTELLIGENZ EINES MACROPUS GIGANTEUS ZIMMERMANN
}

\author{
VON \\ FLORRIE HEUBEL \\ Aus dem Tierpsychologischen I.aboratorium der Königl. Zool. Gesellschaft \\ "Natura Artis Magistra" zu Amsterdam
}

\section{EINLEITUNG}

Vor einiger Zeit wurde mir in dem Garten der Kön. Zool. Ges. „Natura Artis Magistra" zu Amsterdam die Gelegenheit geboten, mich eingehend mit einem Känguruh, Macropus giganteus Zimm., zu beschäftigen. Ich möchte Herrn Dr. J. A. Bierens de HaAn, zu gleicher Zeit auch Herrn A. F. J. PorTIELJE, die meiner Arbeit durch ihre gütige Hilfe sehr gedient haben, meinen aufrichtigen Dank aussprechen. Die Wärter möchte ich auch nicht vergessen, keine Mühe war ihnen zu gross.

Bevor ich über meine Versuchsergebnisse berichte, möchte ich einige Worte sagen über die Art und Lebensweise des Känguruh im allgemeinen. Wenn wir uns die Tiere im Zoo betrachten, so werden wir gleich durch das Unharmonische ihres Körperbaus gefesselt: der Körper nimmt von vorn nach hinten an Umfang zu. Wir sehen, dass das Hinterteil, als der wichtigste Teil für diese Tiere, am stärksten entwickelt ist. Die Bewegung wird fast ausschliesslich durch die Hinterbeine und den Schwanz vermittelt.

Betrachten wir uns ein Tier näher, so bemerken wir an den verlängerten Fusswurzeln lange Zehen, im ganzen vier (der Daumen fehlt). Zwei sind lang, mit grossen Nägeln ausgestattet, die beiden anderen sind verkümmert und zu zwei Krallen verwachsen. BRANDEs bezeichnete diese verkümmerte Doppelzehe treffend als „Putzhändchen”. Beim Kratzen hinter den Ohren werden meist die Hinterbeine gebraucht, wobei besonders die kleinen Krallen (die um $90^{\circ}$ gedreht stehen) benutzt werden. Beim übrigen Kratzen gebraucht das Tier dic Vorderpfoten, die klein, kräftig und handartig gebaut sind. Weiter gebrauchen die Tiere die Vorderpfoten zum Ergreifen der Zweige und zum langsamen Gehen bei der Nahrungssuche. Das Tier setzt dazu die Vorderbeine auf und schiebt die Hinterbeine an ihnen vorbei, so dass sie neben den Vorderbeinen zu stehen kommen. Gleichzeitig stützt es sich dabei auf den Schwanz. Dieser Gang sieht dann aus wie ein langsames Forthumpeln.

Manchmal können wir ein schnelles Sich-hin-und-herbewegen mit dem Ober- 
körper beobachten: das Tier sucht das Gleichgewicht zwischen den Hinterbeinen und dem sich versteifenden Schwanz. Auf diese Weise gelingt es ihm, sich sehr hoch aufzurichten, zuletzt ist nur noch die äusserste Spitze des Schwanzes in den Sand gebohrt. In dieser „Dreifussstellung” (von BreHM so bezeichnet)! rupft es sich die Blätter von den Bäumen. Das Ganze macht den Eindruck von ausserordentlicher Muskelkraft im Hinterteil dieses Tieres.

Wie interessant diese Tiere auch sein mögen, dem Tiergärtner bereiten sie durch ihre sehr grosse Scheuheit manchmal viele Sorgen. Bei allem Ungewohnten zeigen sie sogleich gespannteste Aufmerksamkeit. Die Ohrmuscheln, die vorher spielend hin und her gingen, richten sich jetzt dem Laute zu, die Nüstern haben sich weit geöf fnet und schon im nächsten Augenblick entfernen sich die Tiere eiligst mit grossen Sprüngen. Bemerkenswert ist, dass die Vorderpfoten dabei nie benutzt werden, sie hängen oder liegen gekreuzt dicht an der Brust. Der Schwanz dient bei den Sprüngen als Ruder. Oft habe ich bei dem Stande von ,gespanntem Lauschen" auch ein Trommeln wahrgenommen, verursacht durch die Hinterbeine, die abwechselnd auf den Boden geschlagen werden. Dasselbc finden wir bei Hirschen, Ziegen, Hasen und Kaninchen; die letzteren schlagen ebenfalls mit den Hinterpfoten auf den Boden, während Hirsche und Ziegen mit den Vorderpfoten stampfen.

Wir sehen also, dass das Känguruh ein typisches Beispiel eines S c h r e c kund Fluchttieres ist. Die Flucht und die Möglichkeit, sich schnell entfernen zu können, ist für diese Tiere eine Lebensnotwendigkeit. Nur der sexuelle Kampftrieb kann das Tier zum Angriff oder zur Verteidigung bringen.

Das Ka m p f e n habe ich bei meinem Versuchstier einem Kasuar im benachbarten Käfig gegenüber beobachten können. Wie bekannt „fechten” diese Tiere auf dieselbe Art wie das Känguruh, indem sie auch wie diese die Hinterbeine schnell nach vorne schlagen. Unser junger Kasuar versuchte immer wieder, das Känguruh zu einem Gefecht zu verleiten. Das Känguruh, bei dem der Kampftrieb sich noch nicht ausgesprochen äusserte, war dem Kasuar gegenüber völlig gleichgültig und mied meistens die „Kasuarseite”. Nur einige Male kam es zu einem Gefecht. Beide Gegner schlugen dann schnell und mit Kraft dic Hinterbeine nach vorn. Die Scheidewand des Käfigs zwischen den beiden Tieren bestand unten aus einer hölzernen Schotte $(60 \mathrm{~cm}$ hoch), darüber war Gitter. Die Tiere konnten also nut die Oberkörper von einander erblicken. Das Känguruh hielt sich bei diesem Gefecht mit den Vorderpfoten an einem hölzernen Pfahl fest, der teilweise horizontal an dieser Seite entlang lief.

Häufig sehen wir aber auch im Zoo Känguruhs friedlich mit anderen zusammen mitten im grössten Lärm. Dass hier viele verschiedene Faktoren eine Rolle spielen, ist sicher.

In der Literatur finden wir kaum einige zuverlässige Angaben über diese Tiere. BREHM (I9I2) gibt uns eine ausführliche Beschreibung der verschiedenen Gattungen und deren Leben. Weiter berichtet er uns, dass die Känguruhs im hohen Grade ,geistlose Geschöpfe” sind. Unter den Sinnen dürfte das Gehör obenanstehen. Der Gesichtssinn ist schwächer und der Geruch wahrscheinlich ziemlich unentwickelt. HempelmanN (1926) berichtet ebenfalls, dass Beuteltiere geistlose Geschöpfe sind, sich immer gleich bleiben und meint 
dass sie selbst nach jahrelanger Gefangenschaft die sie pflegenden Wärter kaum von anderen Leuten zu unterscheiden vermögen. Dexler (1909) schlägt die Verstandeskräfte nicht hoch an; den Gesichtssinn bezeichnet er als schlecht, das Gehör dagegen als ausgezeichnet. Er hatte beobachtet, dass „Wallabies" in einer Entfernung von 6-7 $\mathrm{m}$ an dem Platze vorbeigingen, wo er auf dem Anstand sass. Die bis jetzt vorherrschende Meinung über das Känguruh ist also, dass es als ein „stumpfsinniges, dummes Geschöpf bei weitem dem Schafe noch unterlegen" sei.

\section{BESCHREIBUNG DES VERSUCHSTIERES UND EINLEITUNG DER VERSUCHE}

Im Jahre 1935 hat „Natura Artis Magistra” zwei Macropus giganteus Zimm. aus dem Rotterdamer Zoo erhalten. Leider starb das eine sehr bald, das zweite ist aber erhalten geblieben. Diese Tier wurde in Rotterdam am 12. 2. '34 geboren ${ }^{1}$ ). Die Eltern gehörten der aus West-Australien stammenden Subspecies Melangos Gould an. Im September 1935 wurde das Tier (bei uns) krank, es hatte ein Geschwür am Unterkiefer. In jeder Beziehung schien das Tier sich nicht wohl zu fühlen und die Esslust war sehr mässig. Diese Krankheit (die Ursache war dem Arzt unbekannt, für uns auch nicht weiter wichtig) hat ein gutes halbes Jahr gedauert. Anfang Juni sah das Tier wieder gesund und munter aus. Da für das Känguruh während seiner Krankheit besonders gesorgt werden musste, so wurde es in einen Innenkäfig der Fasanerie gesetzt $(3.50 \mathrm{~m}$ lang, $2.80 \mathrm{~m}$ breit), der mit einem Aussenkäfig durch eine Luke in Verbindung stand. Dieser Verschlag eignete sich gut zur Pflege (Sonne, frische Luft und Heizmöglichkeiten). Im Gegensatz zu einem Känguruhhaus, in dem sich die Wärter kaum zeigen, fand hier (wie in der Fasanerie notwendig) im Mittenlauf stets ein reges Hin- und Hergeben der Wärter statt. Für das Publikum blieb dieser Gang aber verschlossen. Das Tier wurde da von einem der besten Wärter gepflegt, und diesen bieden Umständen ist es wohl zu verdanken, dass sich im Wesen des Tieres eine tiefgreifende Veränderung vo'lzogen hatte.

Das Tier, von Natur aus gewöhnt sich sozial anzuschliessen, hat sich hier mit der Umgebung vertraut gemacht. Obwohl es sehr bequem war, sich durch dic Luke nach dem Aussenkäfig zu begeben (wo es auch viel grössere Bewegungsmöglichkeiten hatte), zeigte es sich dort doch. nie. Nur durch die Spuren konnten wir feststellen, dass es abends, wenn kein Mensch sich mehr im Zoo zeigte, und alles draussen ruhig war, dort herumgesprungen war. Es hat sich also dem Milieu des Innenkäfigs angepasst; es hat hier sogar den Wärtern gegenüber in seinem sozialen Drang Befriedigung gefunden und sich ihnen völlig angepasst. Schliesslich wurde es so zahm dass, wenn der Wärter sich

1) Als Tag der Geburt wurde der Moment angenommen, als zum ersten Male der Kopf des jungen Känguruhs im Mutterbeutel gesehen wurde. 
eine Pindanuss in den Mund nahm und sich nach vorn beugte, das Tier diese sogleich mit seinem Mund heraus nahm. Wiederholte ich dasselbe, nachdem das Tier sich nach einigen Tagen an meine Gestalt gewöhnt hatte, so nahm es auch mir die Pindanuss aus meinem Munde. Versuchte aber der Wärter dem Tiere im Aussenkäfig auf dieselbe Art die Pindanuss anzubieten, so sahen wir, dass das Tier, welches sich im Innenkäfig uns beiden (nach der Gewöhnung) sofort zutraulich genähert hatte, sich jetzt erschreckte und sofort flüchtete. Der Wärter wurde also nicht als „Person a n sich” erkannt, sondern nur als ,an den Ort (Innenkäfig) gebundene Person". Durch die Trennung des Wärters von dem bekannten Ort wird die Einheit zerbrochen, das Tier erschrickt und flüchtet. Man fühlt, dass hier noch höchst interessante Gebiete of fenliegen, z. B.: in wie weit diese Ortsgebundenheit etwas Starres ist. Es würde uns aber zu weit führen, hier näher darauf einzugehen.

Früh morgens bekam das Tier sein Futter, bestehend aus Brot, Hafer, Apfelstückchen, Karotten, Tomaten, Bananen und Hafergrütze, des öfteren auch einen Ulmenzweig, den es am meisten liebte und von dem es zuerst die Rinden abknabberte. (Bei den Bananen wird auch zuerst die Schale abgerupft und gegessen.) Es war bemerkenswert, dass das Tier beim Hinlegen nach dem Essen nie versäumte, sich mit den Vorderpfoten Sand über die Hinterbeine zu werfen. Auch wenn es durch eine Fliege gestochen wurde bewegte es zuerst die Haut schnell hin und her, dann aber warf es mit den Vorderpfoten Sand nach dem Fleck, wo die Fliege sass. Wir müssen hier aber gut unterscheiden, ob das Tier diese Handlung ,zielbewusst” oder nur zufällig ausübt. Eine ähnliche Handlung können wir bei den Elefanten wiederfinden, wo sich die Tiere mit dem Rüssel Sand auf den Rücken werfen. Einen biologischen Wert werden beide Handlungsweisen bestimmt haben, ob man hier aber von angeborener oder angelernter Handlung sprechen muss, will ich nicht entscheiden.

Ende Juni 1937 was es mir möglich, mich mit diesem Tieré zu beschäftigen. Die ersten Tage verbrachte ich im Käfig, um das Tier zu mir Vertrauen fassen zu lassen. Ich gab acht, mich äusserst vorsichtig zu benehmen, um das Tier in keiner Weise zu erschrecken.

$\mathrm{Zu}$ meinen Versuchen liess ich mir ein Brett machen (1,10 m lang, 0,30 m breit), das in $0,38 \mathrm{~m}$ Höhe an der kurzen Wand des Verschlages befestigt wurde. Das Futter, das auf das Brett hingelegt wurde, war für das Tier bequem zu erreichen. Da ich nun bemerkte, dass es sich durch meine Anwesenheit im Käfig beeinflussen liess, schien es mir besser, mich ausserhalb des Käfigs aufzustellen und das Futter durch das Fenster auf das Brett hinzulegen.

Die Fragen die ich zu beantworten suchte waren die Folgende:

A. Welche ist beim Känguruh die Bedeutung und die Rangordnung der Sinne?

I. Der Geruchssinn.

2. Der Gesichtssinn.

B. In wie weit erstrebt das Tier bevorzugtes Futter? 
C. Ist die Erinnerung an das bevorzugte Futter so stark, dass es zu einem Suchen danach kommt?

D. Ist es möglich, das Känguruh auf Farbe zu dressieren?

F.. Wie ist es mit seiner Intelligenz bestellt?

Wir wollen jetzt sehen, wie das Känguruh diese Fragen beantwortete.

\section{DIE VERSUCHE}

\section{A. VERSUCHE UBER DIE RANGORDNUNG DER SINNE}

Beim Hinlegen verschiedener Gegenstände (Futter und Schein-Futter) werden diese vermutlich schon von weitem halb olfaktorisch, halb optisch wahrgenommen (das Tier nähert sich, den Kopf dem Futter zugewandt), jedoch beruht die feinere Unterscheidung ausschliesslich auf dem Geruch. Das Tier beschnüffelt die verschiedenen Gegenstände, das Futter wird aufgenommen, das Scheinfutter bleibt liegen.

Der Geruchssinn ist also für die Nahorientierung und das genauere Erkennen des Futters der Wichtigste.

a. Der Geruchssinn.

Um den Geruchssinn untersuchen zu können, musste ich die Möglichkeit ausschalten, dass die anderen Sinnesorgane bei den Versuchen benutzt werden konnten.

$\mathrm{Zu}$ diesem Zweck nahm ich zwei Blumentöpfe und legte sie dem Tiere vor. Nachdem das Tier diese einige Sekunden beschnüffelt hatte, zeigte es kein weiteres Interesse mehr dafür.

I. Versuch:

Zwei umgekehrte Blumentöpfemit $50 \mathrm{~cm}$ Abstand a uf dem Brett.

Ein Topf enthält Futter (Ulmenblatt), der andere ist leer. Nach jedem Versuch wurde das Futter in den anderen Topf getan, das Tier konnte sich also nicht nach einer Seite orientieren.

Bei 4o Versuchen wurde stets der Topf mit dem Futter umgedreht (Abb. I).

Der leere Topf wurde weder verschoben, noch berührt. Das Tier erkannte also das Futter unter dem umgekehrten Topf.

2. Versuch:

Zwei umgekehrte Blumentöpfe, mit $50 \mathrm{~cm}$. Abstand a uf dem Brett und einer 2 cm dicken Sandschicht.

Anfangs machte ich den Fehler, den Sand aus dem Käfig selber zu holen, auf dem seit Monaten die Ulmenzweige gelegen hatten.

Bei diesen 40 Versuchen wurden 3 Fehler gemacht (das Tier drehte also dreimal den leeren Topf um), weiter zeigte es in der Wahl eine kleine Unsicherheit. Offenbar hatte der Sand einen Ulmengeruch angenommen. 
Nachdem ich aber den Fehler mit dem alten Sande entdeckt und den alten Sand durch neuen ersetzt hatte, zeigte das Tier sich in der Wahl sicher. Ein Beweis also, wie scharf das Tier olfaktorisch zu unterscheiden vermag, Bei 40 Proben wurde stets nur der Topf mit dem Futter umgedreht. Auch hier wurde der leere Topf weder umgedreht noch berührt.

\section{Versuch:}

Zwei umgekehrte Blumentöpfe, mit $50 \mathrm{~cm}$ Abstand a uf dem Brett, in einer $2 \mathrm{~cm}$ dicken Sandschicht. Die Löcher der Töpfe waren mit Gips zugemacht.

In beide Töpfe wurde jetzt Futter hineingelegt.

Bei 20 Proben hat das Tier keinen Topf umgedreht oder verschoben. Nach jedem Versuch aber gab ich dem Känguruh ein Ulmenblatt zur Belohnung. Nachdem es beide Töpfe beschnüffelt hatte ohne etwas wahrzunehmen, entfernte es sich, ohne sich weiter um die Gegenstände zu kümmern.

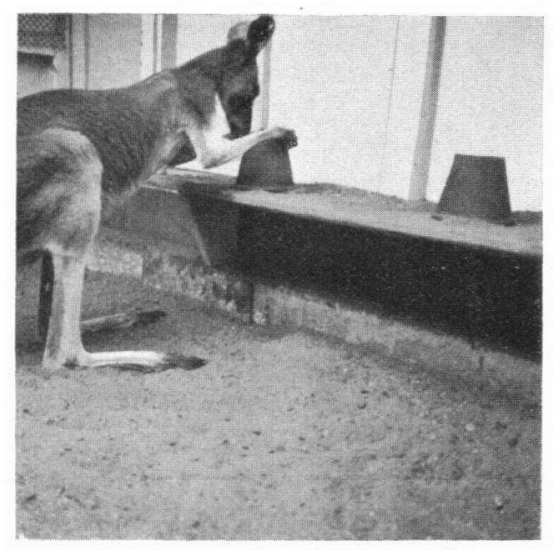

Abb. I. Känguruh wählt den Blumentopf, der über das Futter umgedreht ist.

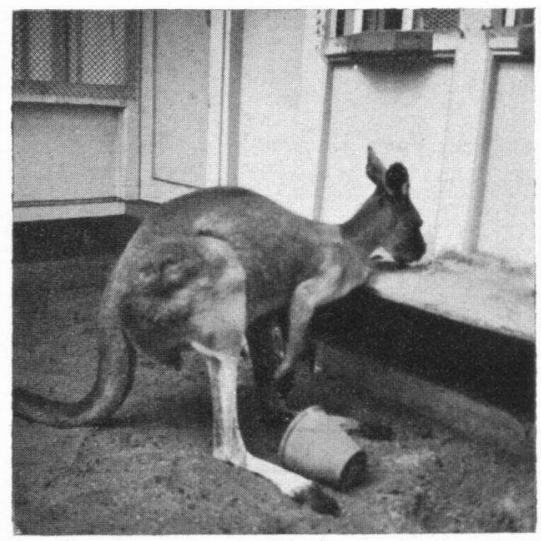

Abb, 2. Känguruh hat den Topf hinuntergeworfen und frisst nun das Futter.

Die Folgerung ist also, dass beim ersten Versuch das Futter mittels des Geruchssinnes durch das obere Loch und durch den unteren Rand des Topfes wahrgenommen werden konnte. Das Tier wählte dabei Ioo\%ig richtig.

Beim zweiten Versuch war die Aufgabe erschwert. Der Geruch des Futters konnte nur noch durch das Loch wahrgenommen werden, da der Topf unten durch den Sand abgeschlossen war. Doch auch hier zeigte das Tier noch eine IOo \% ig richtige Wahl.

Beim dritten Versuch, bei dem der Geruchssinn durch den Sand und das Verstopfen des Loches gänzlich ausgeschaltet wurde, ging das Tier, nachdem es die Töpfe berochen hatte, an ihnen vorüber, obwohl sich Futter darin befand.

Dies beweist also, dass das Känguruh sich erst dann Mühe gab, die Töpfe 
umzudrehen, wenn es auch wirklich Futter olfaktorisch wahrgenommen hatte, sonst zeigte es kein weiteres Interesse.

Der Geruchssinn ist also äussert entwickelt und dementsprechend sehr wichtig für das Känguruh, wie die Versuche uns zeigen.

Interessant ist dabei zu sehen, wie das Tier die Aufgabe löst, den Topf umzudrehen. Nachdem es erst etwas ungeschickt den Topf mit der Schnauze verschieben oder umwerfen wollte, lernte es sehr bald, seine Vorderpfoten hierfür zu benutzen (Abb. I). Dabei umklammerte es den Topf mit beiden Vorderpfoten, schob ihn an den Rand des Brettes und stösste mit der Schnauze gegen den Topf, wodurch er auf den Boden fiel und das Futter zum Vorschein kam (Abb. 2).

\section{b. Der Gesichtssinn.}

Bei meinen vorigen Versuchen haben wir gesehen, dass das Futter bei einem umgekehrten, von oben zugemachten Topf, der auf dem Brett in $2 \mathrm{~cm}$ dicker Sandschicht stak, olfaktorisch nicht mehr wahrgenommen wurde. $\mathrm{Zu}$ diesen Versuchen nehmen wir jetzt zwei Geleegläser ( $13 \mathrm{~cm}$ hoch, Diameter $7 \mathrm{~cm}$ ). In das erste wird nichts, in das zweite ein Ulmenblatt gelegt. Die Gläser werden umgekehrt in I $\mathrm{m}$ Abstand auf das Brett in den Sand gesetzt. Bei jedem Versuch wird Glas und Futter gewechselt.

\section{Versuch:}

Geht das Känguruh gleich auf das Glas mit dem Futter zu, nachdem es Gelegenheit gehabt hat, dieses optisch wahrunehmen?

Sehr bald stellte sich heraus, dass das Tier die Gewohnheit hatte, sich bei jedem Versuch so zu drehen, dass es mit seinem Kopfe genau zwischen die beiden Gläser kam. Aus dieser Lage wurde dann gewählt; das Känguruh war dabei also $50 \mathrm{~cm}$ von den Gläsern entfernt.

Bei 20 Versuchen richtete das Tier sich immer zuerst nach dem Ulmenglas, das leere Glas wurde dabei nicht beachtet, höchstens flüchtig beschnüf felt, nie aber umgeworfen.

In einem Abstande von $50 \mathrm{~cm}$ unterscheidet das Tir also optisch ein leeres Glas genau von einem mit Ulmenblatt gefüllten Glase und wendet sich dementsprechend gleich dem Glase mit dem Ulmenblatt zu.

2. Versuch:

Unterscheidet das Tier zwei Fut terarten, die in Form und Grösse verschieden sind, auf $50 \mathrm{~cm}$ Entfernung?

Auf dem Brette mit Sand werden im Abstande von I m zwei Gläser hingestellt, das eine mit Brot, das andere mit Ulmenblatt. Die Frage ist jetzt : richtet das Tier sich gleich seiner Vorliebe, dem Ulmenblatt zu, wenn es in der Mitte die beiden Futterarten wahrnimmt? Bei verkehrter Wahl (in diesem Falle: Brot), nahm ich schnell das Brot weg, das Tier sah sich dann in keiner Weise belohnt. Es wurde dadurch zur Aufmerksamkeit gezwungen. 
Am ersten Tage der Versuche verhielt das Tier sich fast völlig rechtsläufig und zeigte kein Interesse.

Am zweiten Tage gab es sich mehr Mühe, um das Futter zu bekommen, demnach wurde auch das Brotglas öfters umgeworfen, wodurch es seine Rechtsläufigkeit aber verlor.

Am dritten Tage bekam es aber wieder einen Rückfall und verhielt sich wieder völlig rechtsläufig.

Am vierten Tage fing aber die gute Unterscheidung an. In der Mitte $z$ wischen den Gläsern, schaute es zuerst nach beiden Seiten, um sich dann bei 50 Versuchen 30 mal (also $60 \%$ ) dem bevorzugten Futter zuzuwenden. Am fünften Tag machte es keinen Fehler mehr, roo \% wurde das Glas, das Ulme enthielt, zuerst umgeworfen.

Im ganzen waren also 200 Versuche in 5 Tagen nötig, um Futter, in Form und Grösse verschieden, auf einen Abstand von $50 \mathrm{~cm}$ durch das Tier unterscheiden $z u$ lassen.

Die beigefügte Kurve gibt zur Verdeutlichung ein gutes Bild (siehe Abb. 3). In der Zeichnung ist nur die Wahl bei rein optischer Wahrnehmung gebracht, jede zufällige Wahl ist nicht angegeben worden.

\section{Versuch:}

Unterscheidet das Känguruh Futter bei gleicher Form und Grösse?

Zwei kleine Gläser $(9 \mathrm{~cm}$ hoch, Diameter 4. $\mathrm{cm}$ ) werden umgekehrt auf das Brett in den Sand gestellt. Das eine mit einer Pindanuss, das andere mit einem Stück Brot, beides von gleicher Form und Grösse. (Die Pindanuss wird sehr bevorzugt.)

Bei I5O Versuchen in 4 Tagen hat das Känguruh gelernt, die zwei Futterarten auf eine Entfernung von $50 \mathrm{~cm}$ zu unterscheiden, sich also der Pindanuss zuerst zuzuwenden.

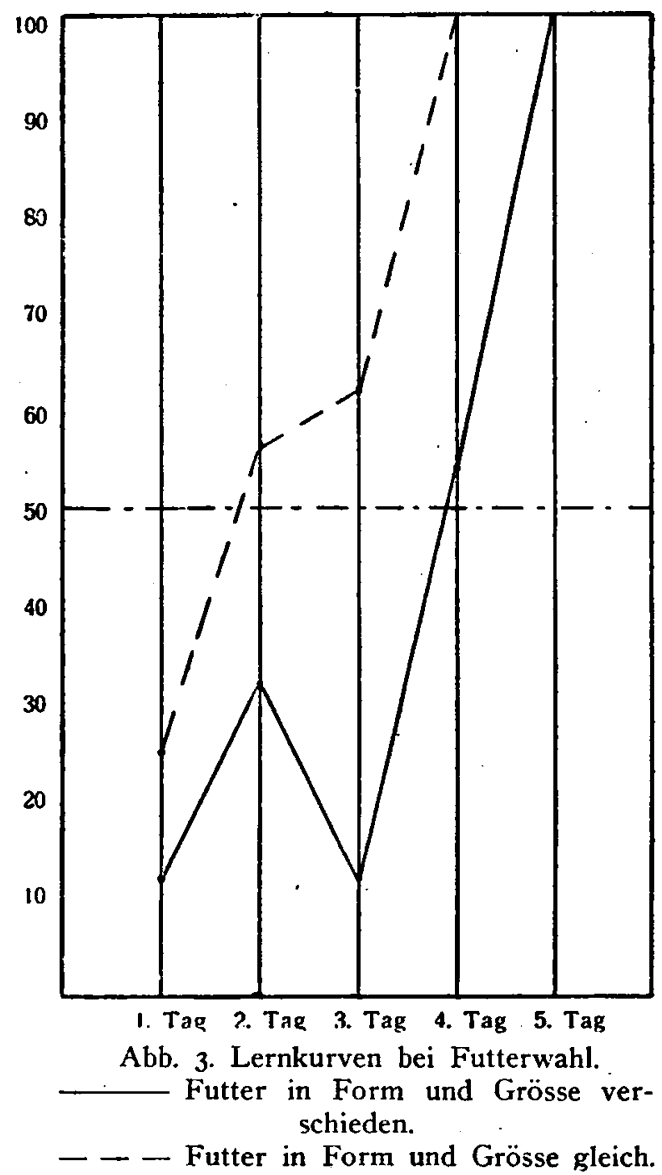

Wenn wir jetzt die Kurven betrachten (siehe Abb. 3), und die beiden Linien vergleichen, so sehen wir, dass das Tier bei einem Futter, welches in Form 
und Grösse gleich ist, einen Tag weniger nötig hatte, als bei Futterarten, welchc ungleich in Grösse und Form sind. Dies mag etwas unbegreiflich sein, denn zwei Futterarten, gleich in Grösse und Form, sind doch schwieriger aus der Entfernung zu unterscheiden. Wenn wir aber die Kurve sehr genau betrachten, so sehen wir, dass das Känguruh bei diesen Versuchen die ersten Tage Mühe hatte, rein optisch wahrzunehmen. Nach dem 4. Tag steigt die Kurve aber bedeutend, und diese Kurve, also bei Futter in Form und Grösse verschieden, läuft fast parallel mit der anderen. Kurz gesagt, nachdem das Tier das Futter rein optisch wahrnimmt, machen die beiden Futterarten (gleich oder verschieden in Form und Grösse) keinen grossen Unterschied mehr aus.

Als Kontrollversuch habe ich beide Gläser, das eine mit Brot, das andere mit Pindanuss, durch Deckel vollkommen geschlossen. Bei der Wahl machte dies aber keinen Unterschied, womit also bewiesen ist, dass das Tier optisch wahrnimmt.

Im allgemeinen warf das Tier die offenen Gläser mit der Schnauze um, steckte die Nase in die Öffnung und holte sich mit der Zunge das Blatt heraus (Abb. 7). Niemals wurden die Vorderpfoten bei dem Herausholen benützt.

Wir können also jetzt mit Sicherheit sagen, dass der Geruchssinn an erster Stelle steht und der Gesichtssinn erst an $z$ weite Stelle kommt.

\section{B. UNTERSUCHUNGEN UBER DAS ERSTREBEN BEVORZUGTEN FUTTERS}

Schon oben sahen wir, dass das Känguruh für einige Futterarten besondere Vorliebe zeigte. Jetzt wollen wir untersuchen, in wieweit das Tier sich selbst besondere Mühe gibt, dieses beliebte Futter zu erlangen. Es hat beim Futtersuchen die Gewohnheit, verschiedene Futterarten zu beschnüffeln und dann das bevorzugte zuerst zu verzehren.

I. Versuch:

$\mathrm{Z}$ wei Futterarten nebeneinander a uf dem Brett. (Ulmenblatt -.. Brot.)

Das Känguruh beschnüffelt beide Futterarten. Bei 25 Versuchen wird an erster Stelle aber immer das bevorzugte Futter (Ulmenblatt) genommen, danach erst wird das Brot gefressen.

\section{Versuch:}

Zwei Futterarten in einem Abstand von $30 \mathrm{~cm}$ auf dem B ret t. (Ulmenblatt - Brot.)

Bei 25 Versuchen (wobei die Stelle des Futters immer gewechselt wird) nimmt das Tier das beliebtere Futter (Ulme) 25 mal als erstes auf. Zwar wird im Anfang das Brot an erster Stelle beschnüffelt, aber nie gefressen. Sehr bald wendet das Tier sich aber direkt der Ulme zu, und das Brot wird erst an zweiter Stelle verzehrt. Wenn ich statt Ulme aber zwei Stükchen Brot hinlege, so frisst das Känguruh das nächstliegende zuerst auf. 
Wir sehen also, dass das Känguruh das weniger beliebte Futter liegen lässt, um sich dem besseren zuzuwenden. Das Tier hat dabei seine Gewohnheit aufgegeben, jeden Gegenstand zuerst zu beschnüffeln, denn meist ging es sofort zum Ulmenblatt (olfaktorisch!).

\section{Versuch:}

Zwei Futterarten, in einem Abstand von I Meter, auf dem Brette.

Das Känguruh wendet sich nun nicht sofort dem bevorzugten Futter zu, sondern es fällt in die Rechtsläufigkeit zurück und infolgedessen werden beide Futterarten abwechselnd aufgenommen. Der Ulmengeruch ist also auf diese Entfernung zu schwach, um anziehend zu wirken.

Die Frage ist nun:

Ist es mögli ch, be i dieser Entfernung, das Känguruh zu einem Wählen des bevorzugten Futters auf optischer Wahrnehmung zu bringen?

Die Stelle der beiden Futterarten wird nach jedem Versuch gewechselt.

Die Antwort auf diese Frage gibt uns Abb. 4. Da sehen wir zwei Linien: die

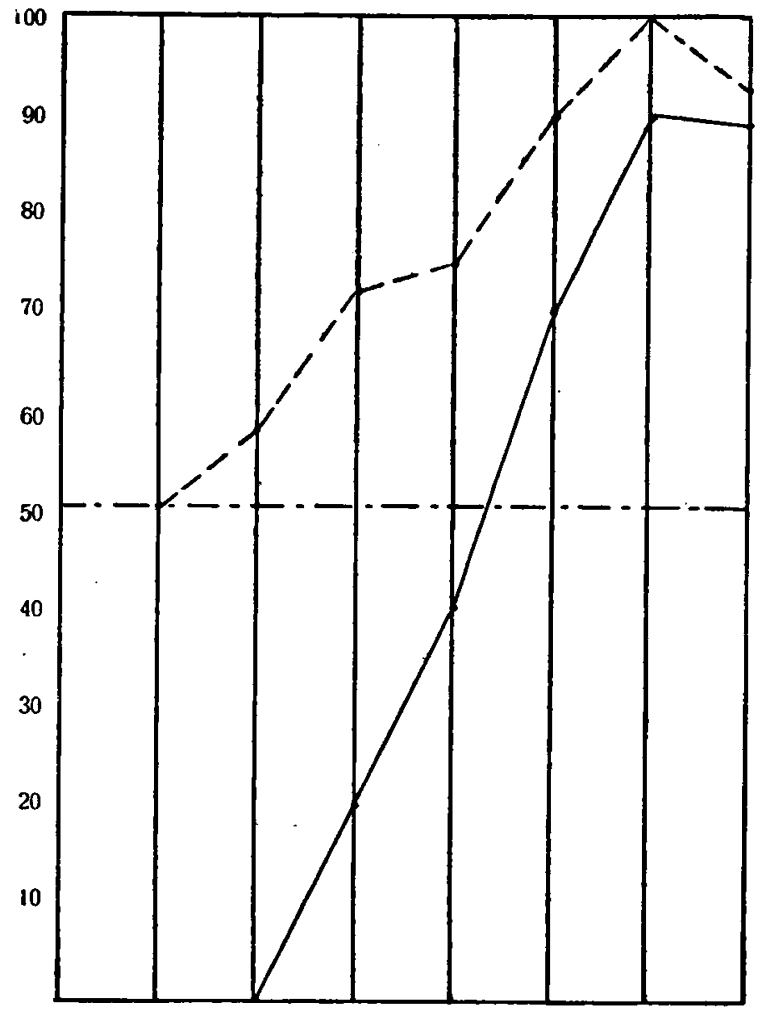

1. Tag 2. Tag 3. Tag 4. Tag 5. Tag 6. Tag 7. Tag

Abb. 4. Wahl des bevorzugten Futters.

- - Wahl des bevorzugten Futters im allgemeinen.

Wahl des bevorzugten Futters auf optischer Wahrnehmung. gestrichelte zeigt uns, wie oft (im ganzen) pro Tag das Känguruh das bevorzugte Futter als erstes genommen hat; die gezogene, für uns durchaus die wichtigste, gibt die Häufigkeit der Wahl des bevorzugten Futters bei optischer Wahrnehmung an.

Hinzufügen möchte ich, dass ich nur dann von Wahl auf optischer Wahrnehmung spreche, wenn das Tier in der Mitte deutlich nach beiden Seiten blickt, seine Wahl trifft und sich dann sofort dem bevorzugten Futter zuwendet.

Bei 215 Versuchen in 6 Tagen können wir also sagen, dass das Tier gelernt hat, auch in dieser Entfernung das bevorzugte Futter durch optische Wahr- 
nehmung zu unterscheiden. Wir sehen noch einmal, dass das Tier am leichtesten olfaktorisch wahrnimmt, und nur, wenn es gezwungen wird optisch wahrzunehmen, dieses mit Mühe lernt und so deutlich dem bevorzugten Futter zustrebt.

C. IST DIE ERINNERUNG AN DAS BEVORZUGTF. FUTTER SO STARK, DASS ES ZU EINEM SUCHEN DANACH KOMMT?

I. Versuch:

Zwei Tröge (I $7 \mathrm{~cm} \mathrm{hoch,} \mathrm{Diameter} 30 \mathrm{~cm}$ ), der eine Ulmen, der andere Brotenthaltend, werden in $30 \mathrm{~cm} \mathrm{Ab-}$ stand auf das Brett gestellt.

Am ersten Tage zeigte das Känguruh sich rechtsläufig, beide Futter wurden abwechselnd ohne Unterscheid an erster Stelle gefresen. Am nächsten Tage aber, wenn das Brot als erstes gefressen war, richtete sich das Tier gleich dem anderen Trog zu, um auch noch das Ulmenfutter zu erhalten. Es erinnerte sich also wahrscheinlich des besseren Futters. Am dritten Tage wurde bei 50 Versuchen 42 mal (84 \%) Ulmen, 8 mal ( I6\%) das Brot gesucht. Am 4. Tage wurde

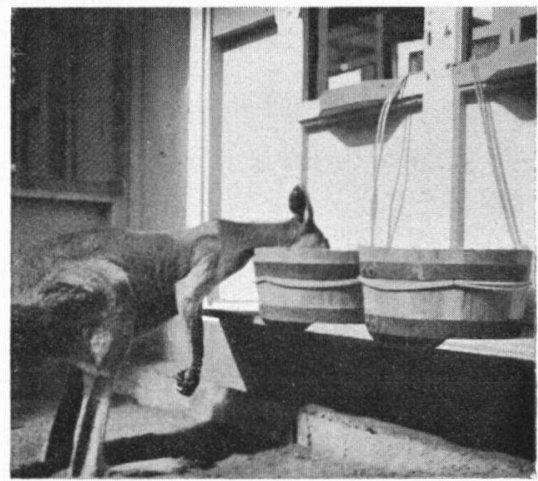

Abb. 5. Känguruh nimmt das Futter aus dem Trog. bei 40 Versuchen 100 \% das bevor-

zugte Futter gewählt; dabei sah das Tier 26 mal (65\%) zuerst nach dem weniger beliebten Futter, nahm jedoch das bessere (Abb. 5).

Deutlich sehen wir hier, dass die Erinnerung an das bevorzugte Futter so stark ist, dass das Känguruh danach sucht.

2. Versuch:

Ist die Vergrösserung des Abstandes auf I m von Einfluss? Zuerst verfiel das Känguruh bei diesem weiteren Abstande wieder in Rechtsläufigkeit. Bei 25 Versuchen werden Brot oder Ulme abwechselnd an erster Stelle aufgefressen, dann aber kam das wirkliche Suchen nach dem bevorzugten Futter. Bei den nächsten 50 Versuchen in 2 Tagen wurde jeweils nur das Ulmenblatt, also Ioo \%ig gefressen.

Hier sehen wir also, dass die Erinnerung den Ausschlag gibt und die Entfernung zwischen den beiden Trögen eine untergeordnete Rolle spielt.

3. Versuch:

Erhält sich die Erinnerung einige Tage?

Nach zwei Tagen wurden dieselben Versuche wiederholt. Bei I50 Versuchen in zwei Tagen suchte das Känguruh I28 mal ( $85,5 \%)$ das bevorzugte Futter. Die Erinnerung an das bevorzugte Futter blieb also in diesen Zeit erhalten 
4. Versuch:

Wird auch noch nach dem besseren Futter gesucht, wenn die Aufgaben erschwert werden?

Auf beide Tröge, Ulme und Brot

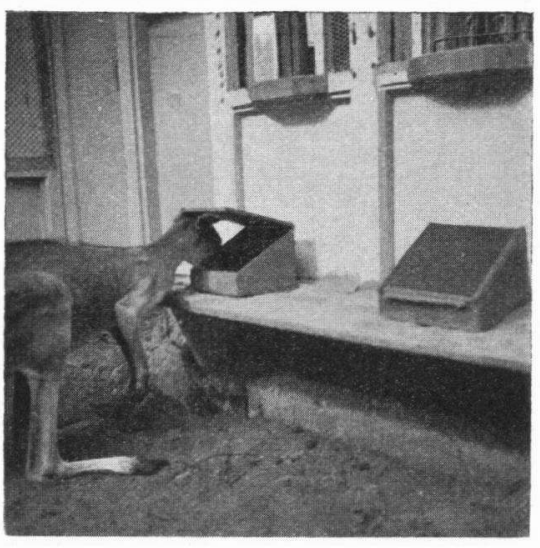

Abb. 6. Känguruh öf fnet den mit einer weissen Platte markierten Kasten. enthaltend, werden jetzt Deckel (Diameter $35 \mathrm{~cm}$ ) gelegt, wodurch das Futter weder gesehen noch gerochen werden kann.

Bei 25 Versuchen in zwei Tagen wurde 25 mal (also Ioo \%) das bevorzugte Futter gesucht, wobei der Deckel mit der Schnauze verschoben wurde. Sogar die Mühe, den Deckel mit der Schnauze wegzuschieben, war also nicht zu gross, um das Tier erstreben zu lassen, das bevorzugte Futter zu erreichen. Das Brot wurde jetzt aber nicht mehr an zweiter Stelle gefressen, die Mühe dafür war wohl zu gross.

\section{IST ES MÖGLICH, DAS KÄNGURUH AUF „WEISS” GEGEN „SCHWARZ” ZU DRESSIEREN?}

I. Methode:

Zwei Kästen, eins weiss, eins schwarz markiert, stehen in einem Abstande von 6 o cm a u dem Brette.

Dic Kästen haben eine Grundfläche von $18 \times 22 \mathrm{~cm}$, und sind an der Hinterseite $16 \mathrm{~cm}$ hoch. Auf den abfallenden Deckel, der etwas hervorspringt und dadurch leicht aufklappbar ist, werden die schwarzen oder weissen Platten $(20 \times 20 \mathrm{~cm}$ ), aufgeschoben (Abb. 6). Jeden Tag wird das Futter in den anderen Kasten gelegt, bei jedem Versuch auch die Stellung der Kästen geändert (r-1-r-1-r-r-1-1-r-1). Seitendressur war also völlig ausgeschlossen.

Die ersten Tage war das Känguruh völlig rechtsläufig, dabei wurde der weisse Kasten (Ulmenblatt enthaltend) geöffnet, und der schwarze (leer) nüï beschnüffelt.

Am 4. Tag war die Rechtsläufigkeit teils überwunden, das Tier zweifelte bei seiner Wahl. Am nächsten Tage wurde es noch unsicherer. Beide Kästen wurden jetzt geöffnet, 5 mal bei 50 Versuchen aber hatte es durch optische Wahrnehmung sogleich weiss genommen. Am nächsten Tage verhielt das Tier sich ähnlich, während es am 7. Tage wieder in Seitenstetigkeit zurückfiel.

Nach 360 Versuchen an 7 Tagen habe ich diese Versuche aufgegeben.

Deutlich war zu bemerken, dass das Känguruh nicht optisch, sondern olfaktorisch wahrnahm. Durch diese Versuche ist also noch nicht bewiesen, dass 
Weiss-Dressur bei dem Känguruh unmöglich ist, sondern eher, dass meine Arbeitsmethoden noch nicht vollkommen waren. Auch jetzt hat uns das Känguruh aber wieder deutlich gezeigt, dass der Geruchssinn bei weitem von führender Bedeutung ist.

2. Methode:

Zwei Geleegläser (I $3 \mathrm{~cm} \mathrm{hoch,} \mathrm{Diameter} 7$ ) in $60 \mathrm{~cm} \mathrm{Ab-}$ stand auf dem Brett in $2 \mathrm{~cm}$ dickem Sande.

Schon früher ist gezeigt worden, dass bei dieser-Methode der Geruchsinn ausgeschlossen ist. Das eine Glas wurde mit Ripolin schwarz, das andere weiss angemalt.

Zuerst zeigte das Känguruh sich rechtsläufig, hatte wenig Lust an der Arbeit. Bei dieser Methode war das Wahrnehmen von Futter sowohl optisch wie olfaktorisch ausgeschlossen. Schon am zweiten Tage zeigten das Känguruh keine Rechtsläufigkeit mehr, eher Zweifel, nach welcher Seite es sich zuerst richten sollte. Des öfteren ging es an dem schwarzen Glase vorbei, ohne er weiter zu beriechen. I4 mal ( $28 \%$ ) bei 50 Versuchen nahm es den weissen Topf optisch wahr.

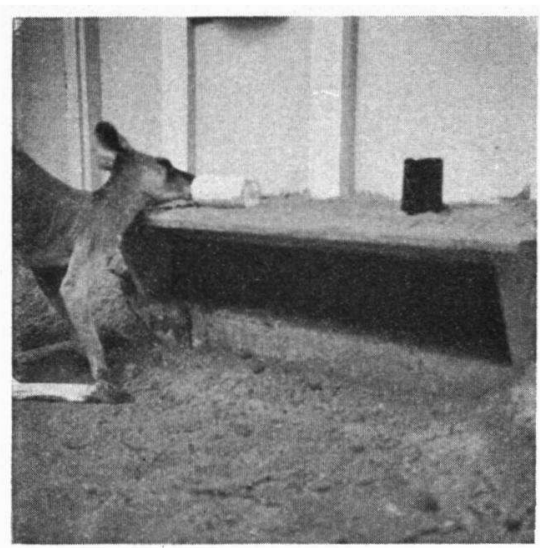

Abb. 7. Känguruh wirft das weisse Glas um und nimmt das Futter.

Am dritten Tage richtete sich das Tier bei 60 Versuchen 38 mal $(63,46 \%)$ nach weiss, $6 \mathrm{mal}$ (10,02 \%) wurde schwarz umgeworfen und $16 \mathrm{mal}$ $26,72 \%$ ) wurde an schwarz vorüber gleich nach weiss gegangen.

Am nächsten Tage richtete es sich bei 50 Versuchen $45 \mathrm{mal}(90 \%)$ gleich nach dem weissen Glase.

Wir können also jetzt sagen, dass das Känguruh sich in 4 Tagen bei 210 Versuchen völlig auf weiss eingestellt hatte. Als wir dieselben Versuche nach einigen Tagen wiederholten, so sahen wir, dass es sich bei 25 Versuchen $21 \mathrm{mal}(84 \%)$ gleich dem weissen Glase zuwendete. Die Erinnerung, sich nach weiss zu richten; blieb also einige Tage erhalten (Abb. 7).

Als Kontrollversuch habe ich schwarzes Papier um das weisse, und weisses Papier um das schwarze Glas gewickelt. Würde das Känguruh olfaktorisch statt optisch gewählt haben (meine Hände gebrauchte ich sowohl zum Umdrehen der Gläser als auch zum Abrupfen der Blätter), so hätte es sich jetzt bei der W'ahl dem Schwarz umwickelten Glase zuwenden müssen. Beide Gläser enthielten je ein Ulmenblatt.

Bei 25 Versuchen richtete das Känguruh sich bei einem Abstande von I $\mathrm{m} 24 \mathrm{mal}(96 \%)$ gleich dem weissen $2 u$. 
Damit ist also bewiesen, dass das Känguruh bei einem Abstande von ungeIähr einem Meter weiss von schwarz unterscheidet und sich dementsprechend gleich danach richtet. In 4 Tagen (bei völligem Ausschalten des Futtergeruchs) ist bei 210 Versuchen das Känguruh vollkommen auf weiss dressiert worden. Die Erinnerung an weiss blieb einige Tage erhalten.

Wir sehen also, dass das Känguruh in kurzer Zeit auf weiss dressiert werden kann. Es interessierte mich, die erste Methode des Kastenversuches nochmals zu wiederholen, da auch die verschiedenartigen Formen der Objekte bei der Wahl von Bedeutung sein könnten.

Zweifellos fallen zwei Gläser (weis-schwarz) mehr auf, als zwei Kästen mit weissem oder schwarzem Deckel. Diese Kästen liess ich besser schliessbar machen, so das der Deckel genau in die Öffnung past, also wenig oder keinen Duft hinauslässt. Ausserdem rieb ich die Seiten vorher mit Ulmenblättern tüchtig ein.

\section{Methode:}

Zwei Kästen (weiss-schwarz) in einem Abstand von $60 \mathrm{~cm} \mathrm{auf} \mathrm{dem} \mathrm{Brett.} 100$

Anfangs zeigte das Tier sich rechtsläufig, 90 danach unsicher bei der Wahl, um am nächsten Tag sich zu $67 \%$ gleich nach weiss zu richten. Am dritten Tage wurde bei 50 Versuchen $3 \mathrm{I}$ mal $(62 \%)$ weiss gewählt, I6 mal (32\%) wurde zuerst an schwarz entlang gegangen, nur $3 \mathrm{mal}$ $(6 \%)$ wurde schwarz an erster. Stelle geöffnet. Am 4. Tage richtete es sich bei 70 Versuchen bei einem Abstande von I $\mathrm{m} 54 \mathrm{mal}$ $(77,22 \%)$ gleich dem weissen zu, I4 mai $(20,02 \%)$ wurde dabei zuerst an schwarz entlang gegangen, 2 mal $(2,86 \%)$ wurde schwarz geöf fnet.

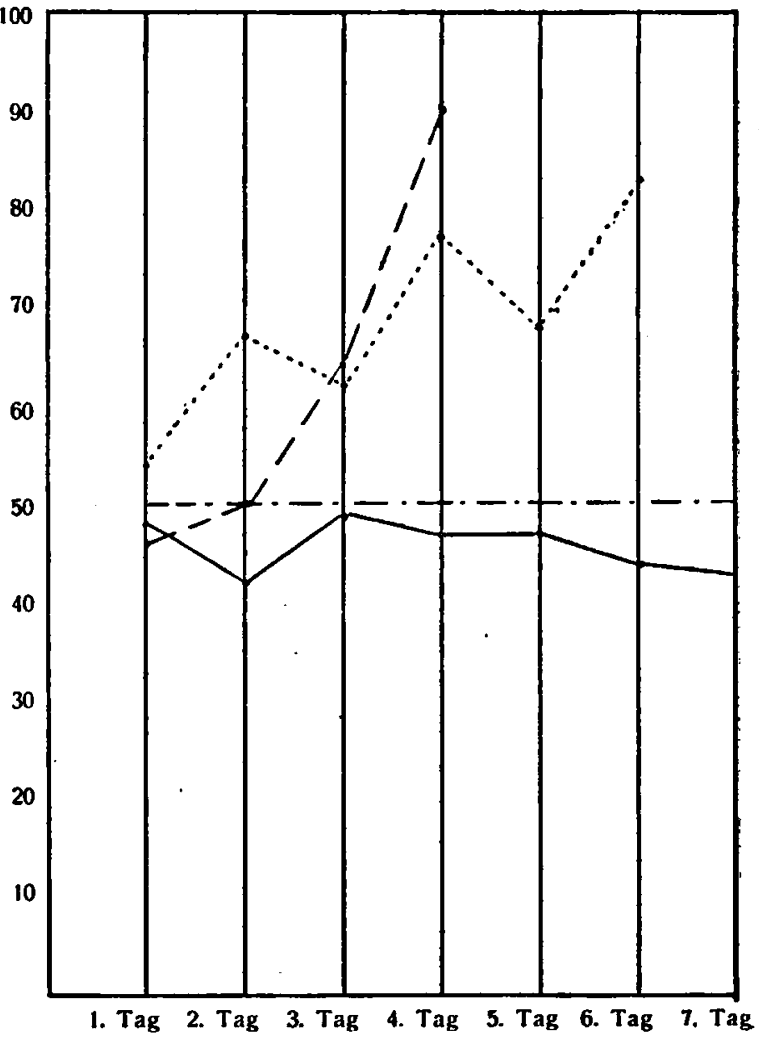

Abb. 8. Verlauf der Weiss-schwarz-Dressur.

- - I. Methode.

.......... 3. Methode. 
Die zwei nächsten Tage richtete es sich meist gleich dem weissen Kasten $\mathbf{z u}$, bei einem Abstand von $\mathrm{I}^{\mathrm{I} / 2}$ Metern.

Zur Kontrolle habe ich das Ulmenblatt mit geruchlosem Futter (Zucker) verwechselt. Auch jetzt wählte das Känguruh bei 20 Versuchen 20 mal (also $100 \%$ ) gleich den weissen Kasten.

Es ist hierdurch also bewiesen, dass sich das Känguruh auch mit der Methode mit zwei Kästen und mit Ausschaltung des Futtergeruches auf weiss dressieren lässt.

Besehen wir uns jetzt die Kurven der Abb. 8 so können wir leicht den Dressurverlauf der drei Methoden verfolgen.

Das Öffnen der Kästen wird immer mit der Schnauze ausgeübt (siehe Abb. 6). Beim Fressen ruht der Deckel dann auf seinem Kopfe. Nie habe ich beobachtet, dass das Tier seine Vorderpfoten hierzu benutzte, nur in sehr seltenen Fällen wurde der Kastenrand mit den Pfoten festgehalten, um weiteres Wegschieben der Kästen zu verhinderen.

Vergleichsweise war es für mich interressant, die Weiss-Dressur nach der ersten Methode mit einem Tiere zu wiederholen, das im Gegensatz zu dem Känguruh mehr optisch als olfaktorisch wahrnimmt. Eine junge Ziege (ein halbes Jahr alt) gebrauchte ich zu diesem Zweck.

Auf einer Kiste ( $30 \mathrm{~cm}$ hoch) wurden die. uns schon bekannten schwarze und weisse Kistchen hingestellt. Die Ziege, auf $3 \mathrm{~m}$ Abstand entfernt, war an einem Strick festgebunden. Bei jedem Versuch wurde sie freigelassen (der Strick gelockert). Bei einem Abstand von I $\mathrm{m}$ richtete sie sich bald gleich dem weissen Kästchen zu.

Am ersten Tage war das Tier völlig rechtsläufig (50\%:50\%). Am zweiten Tage richtete sie sich bei 50 Versuchen $32 \mathrm{mal}(64 \%)$ auf weiss; am dritten Tage 44 mal (88\%) bei 50 Versuchen. Am 4. Tage hat das Tier sich $50 \mathrm{mal}(100 \%)$ auf weissi eingestellt. Also in 4 Tagen hat es die „Weissdressur” vollständig gelernt ( $\mathrm{Zu}$ vergleichen ungefähr mit der 2. Methode bei dem Känguruh.)

\section{E. VERSUCHE ÜBER DIE INTELLIGENZ DES KÄNGURUHS}

Im allgemeinen verstehen wir bei den Tieren unter Intelligenz das Vermögen, erlebice Eindrücke als Erfahrung aufzubewahren und sie bei späteren Gelegenheiten zu verwerten. Schon in den ersten Lebenstagen erhalten Tier und Mensch Eindrücke von Gegenständen und Situationen, denen sie begegnen. Das junge Tier saugt zuerst an jedem Gegenstand, lernt jedoch bald aus Erfahrung die Saugwarze der Mutter von anderen Gegenständen zu unterscheiden. Wenn ein junger Vogel zuerst alle Gegenstände bepickt, so lernt er bald den Unterschied zwischen Stein und Futter. Ein junger Vogel lernt in einem Vogel, der auf dem Nestrande sitzt und Futter im Schnabel hält, das Mutterbild sehen, während alles andere, das nicht dieser Haltung entspricht, ihm „fremd" erscheinen bleibt.

Alle diese Erfahrungen haben eine gewisse Bedeutung für das Tier. Jetzt 
kann es aber sein, dass diese bei einigen Tieren ungeändert bleiben, bei anderen aber diesen Erfahrungen etwas Neues, nicht mehr an den Ort Gebundenes hinzukommt. Das Tier geht einen Schritt weiter. Wenn die Pelikane im Zoo ein durch Gitter abgesperrtes Stück sehen, an einer Seite geöffnet, dann fürchten sie es, nicht wegen des Gitters an sich, sondern durch die Erfahrung, früher einmal in ähnlicher Lage gefangen worden zu sein. Wenn wir aber einen Fischreiher von der einen Aalreuse zur anderen fliegen sehen, um Fische zu räubern, so sehen wir, dass das Tier das nur tut, weil es einmal die Erfahrung gemacht hat, bei einer dieser Aalreusen bequem Fische zu erobern, und nun alle Netze absucht.

Alle diese Beispiele gehören zur niedrigen Intelligenz. Wenn wir nun sehen, dass das Känguruh sich einen Deckel mit der Schnauze wegschiebt wenn es sich Futter aus dem Trog sucht, dieses aber auch mechanisch ausübt, wenn zufälligerweise das Futter auf dem Deckel liegt, so müssen wir dies sehr bestimmt als „niedrige Intelligenz” bezeichnen.

Durch seine Anpassung an die Umgebung und den Menschen, ist seine angeborene Natur aber mit neuen Erfahrungen bereichert worden, die unter normalen Umständen in der Natur sich nie entwickelt hätten. Wir sehen also, dass verschiedene Gegenstände durch Erfahrung eine neue Bedeutung für das Tier bekommen können. Wurden im Anfang Gegenstände nur dann umgedreht, wenn olfaktorische oder optische Wahrnehmung damit verbunden waren, so hat die Erfahrung es jetzt gelehrt, auch ohne diese sinnliche Eindrücke zu handeln.

In früheren Versuchen mit verschiedenen Tieren bei welchen untersucht wurde in wie weit die Tiere noch suchen nach Futter das vor ihren Augen versteckt wurde, hat Bierens de HaAN (1932) diese in 4 Gruppen eingeteilt, je nachdem

A. die Person des Experimentators als Versteck benutzt wird,

B. ein Gegenstand umgestülpt ist,

C. ein Gegenstand geschlossen ist und

D. das Futter ausserhalb des unmittelbaren Bereiches des Tieres versteckt worden ist.

Bierens de HaAn zeigte uns, dass in dem Suchen nach nicht direkt wahrnehmbares Futter ein Intelligenzfaktor steckt. Sicher ist also daher, dass ein Tier, das unter bestimmten Umständen das Futter noch sucht, sich intelligenter benimmt als dasjenige, welches nicht sucht.

Bei dem Känguruh sahen wir schon bei den oben beschriebenen Versuchsergebnissen, dass es das Futter nicht suchte, wenn es olfaktorisch oder optisch nicht mehr wahrzunehmen war. Nur durch Erfahrung konnte es dazu übergehen, die Gegenstände die Futter enthielten, zu öffnen oder umzudrehen.

Die Resultate dieser Versuche mit dem Känguruh können wir also nicht ganz mit denen von BIERENS de HAAN bei anderen Tieren gleichstellen. 
Ergebnisse der Versuche:

A. Die Person des Experimentators selbst wird als Versteck benützt.

a. H a ndöf f nungsversuch.

Das Futter wird vor den Augen des Tieres in die Hand genommen und diese, wenn das Tier zugreifen will, geschlossen.

b. Ta s chenversuch.

Dem Tiere wird das Futter vorgehalten und dieses dann, wenn es zugreifen will, vor seinen Augen in die Tasche des Experimentators fallen gelassen.

Beide Aufgaben werden von dem Känguruh leicht gelöst. Im ersten Falle steckt das Tier sogleich seine Schnauze in die Hand, um das Fıtter zu greifen. Im zweiten Fall schnüffelt es an der Tasche, steckt dann seine Schnauze hinein, schnüffelt und holt sich dann endlich mit Mühe das Futter heraus. Manchmal werden auch die Vorderpfoten zum öffnen der Tasche gebraucht.

Das Futter wird in beiden Fällen von dem Tiere olfaktorisch wahrgenommen.

Die zwei nächsten Versuchsergebnisse, nämlich :

B. Versuche, bei welchen ein Gegenstand umgestïlpt worden war,

c. Blumentop fversuch,

C. Versuche, bei welchen ein Gegenstand geschlossen worden war,

d. Käst e n versuch

sind schon bei den früheren Versuchen am Känguruh ausgeübt worden. Nachdem der Futtergeruch mit diesen Gegenständen in Verbindung gebracht worden war, kostete es dem Tier keine besondere Mühe, das Futter zu erlangen.

D. Das Futter ist ausserhalb der Reichweite des Tieres versteckt niedergelegt.

e. Kisten, in Verbindung mit Bindfaden auf einem T is ch.

Das Futter wird vor den Augen des Tieres in eine Kiste geworfen, die ausserhalb der Reichweite des Tieres auf einem Tisch steht, aber mittels eines angebundenen Bindfadens, dessen freies Ende im Bereich des Tieres auf dem Boden liegt, herangezogen werden kann.

Die Frage ist nun, ob das Tier es versteht, das versteckte Futter auf diesem indirekten Wege heranzuholen. Direkt konnte das Tier auch von der Seite das Futter nicht erreichen, es musste also den Bindfaden benutzen.

Das Känguruh gab sich jedoch überhaupt keine Mühe, das Futter zu erreichen, sogar dann nicht, wenn das Futter in seinem Gesichtsfelde sichtbar lag. Nur einmal wurde ein Sprung ausgeübt, wobei das Tier plötzlich auf dem Tisch landete und seine Mühe dadurch belohnt sah, das Futter jetzt ruhig auffressen zu können.

Der Bindfadenversuch selber fiel also vollkommen negativ aus.

f. Fut terbindfadenversuche $n$ a ch oben. 
Das Futter wird vor den Augen des Tieres an einen Bindfaden gebunden und dann gleich in die Höhe gezogen. (Der Faden läuft über eine Rolle.) Jetzt hängt das Futter ausser Reichweite des Tieres, das freie Ende des Fadens hängt aber herunter und ist im Bereich des Tieres.

Die Frage ist, ob das Tier das Futter auf diesem indirekten Wege sich heranholen kann.

Est nachdem das Futter olfaktorisch wahrgenommen ist, wird es heruntergezogen und aufgefressen.

Der Versuch muss also negativ gedeutet werden.

Zusammenfassend können wir sagen, dass das Känguruh nicht imstande war, die Aufgabe, das Suchen nach nicht-wahrnehmbarem Futter, zu lösen. Nur durch Erfahrung hatte das Tier gelernt, die früher benutzten Gegenstände direkt zu öffnen oder umzudrehen. Bei den neuen Versuchen (Handöffnen, Taschenversuch und Versuchen, ausserhalb Reichweite verstecktes Futter zu bekommen) wurden die Aufgaben nur bei olfaktorischer Wahrnehmung gelöst.

Die Intelligenz ist also nicht hoch bei dem Känguruh, dass aber diese Versuche überhaupt mit dem Tiere möglich waren, hätte man sich vielleicht kaum denken können.

\section{F. VERSUCHE UBER DIE KRAFT DES KÄNGURUHS}

Zuletzt will ich noch über Versuche berichten, die Aufschluss geben können über die Kraft, die das Känguruh in den Vorderpfoten besitzt.

Ein Tau lief über zwei Rollen. An demi einen Ende war ein Ulmenzweig, an dem anderen ein Gewicht aufgehängt.

Die Irrage war jetzt:

Ist das Tier imstande, I kg Gewicht mit seinen Vorderpfoten hochzuziehen?

I. Versuch:

Das Tier greift das Tau, mit Querlatten gesichert, umklammert diese zum besseren Ergreifen abwechselnd mit beiden Vorderpfoten und zieht sich auf diese Weise das Futter heran (Abb. 9a). Beim Fressen hält es sich mit einer Vorderpfote das Tau fest, die andere benutzt es zum Festhalten des Futters.

2. Versuch:

Das Tier setzt sich in die Dreifuss-Stellung (Abb. 9b), zieht das Tau schnell an und hält mit einer Vorderpfote das Futter fest.

Bei 2 Kilo Gewicht.

I. Versuch:

Dreifuss-Stellung. Die äusserste Spitze des Schwanzes bohrt sịch in den Sand. Dann wird das Tau heruntergezogen, das Futter gefressen.

2. Versuch:

Das Känguruh bemüht sich, das Futter heranzuholen, verliert sein Gleich- 
gewicht, fängt aber ohne Schrecken von neuem an, holt sich das Futter heran und hält mit einer Pfote das Futter fest.

Bei $3 \mathrm{Kilo} \mathrm{Gewicht.}$

\section{Versuch:}

Das Tier versucht, sich das Futter heranzuholen, das gelingt ihm aber nicht Jetzt hängt es sich mit dem ganzen Körper an das Tau, endlich wird das Futter erreicht, mit einer Vorderpfote wird dann das Tau festgehalten.

\section{Versuch:}

Das Tier wird fast gänzlich durch das 3 Kilo-Gewicht vom Boden hochgezogen, erreicht nach grosser Mühe endlich das Futter.

3 Kilo ist also das Höchstgewicht, dass das Kängeruh noch imstande ist, mit
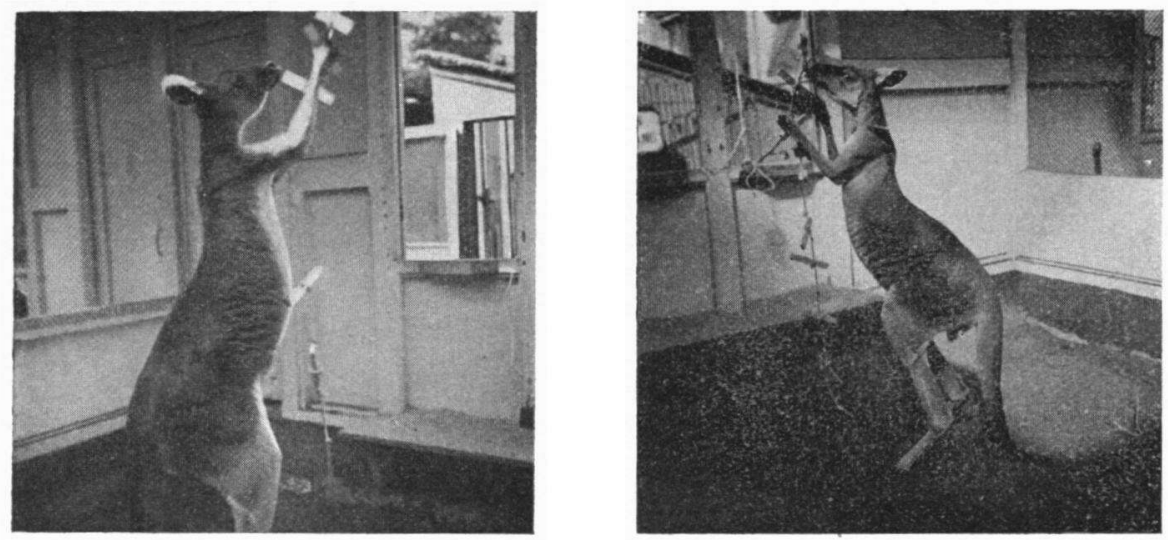

Abb. 9. Känguruh zieht ein über zwei Rollen laufendes und mit einem Gewicht beschwertes Tau hinunter. a. Das Tau wird mit den Vorderpfoten angefasst. b. Das Tier setzt sich dazu in die Dreifuss-Stellung.

den Vorderpfoten hochzuziehen. Bemerkenswert ist dabei, dass, wenn das Tier das Futter erreicht hat, es das Tau mit einer Pfote festhält. Ich betrachte dies als eine ziemlich gute praktische Leistung.

\section{ZUSAMMENFASSUNG}

Bei einem Känguruh, Macropus giganteus Zimm., wurde die Rangordnung der Sinne untersucht. Es stellte sich heraus, dass der Geruchssinn bei weitem überwiegend war und der Gesichtssinn erst an zweiter Stelle kam.

Weiter wurde das Erstreben bevorzugten Futters und die Erinnerung untersucht. Es zeigte sich, dass das Tier sogar bei erschwerten Aufgaben beides positiv löste.

Weiss-schwarz-Dressur wurde in 3 verschiedenen Methoden ausgeübt: Bei 
völliger Ausschaltung des Geruchssinnes wurde in 4 Tagen die Weiss-Dressur erlernt.

Zum Schluss ist die Intelligenz untersucht worden. Sehr hoch ist diese nicht anzuschlagen, aber „stumpfsinnig” sind die Tiere allerwenigst.

\section{LITERATUR}

Bierens de HaAn, J. A.: Uber das Suchen nach verstecktem Futter bei einigen Procyoniden und einem Eichhörnchen. Z.f. vergl. Physiol., 17, 1932.

BReHM, A.: Tierleben (herausgegeben von O. zur Strassen), 1912.

DexLER, H.: Australische Reisebriefe. Lotos, 57, Ig09.

Hempelmann, F.: Tierpsychologie vom Standpunkte des Biologen, Leipzig, 1926. 